\title{
DIAGNÓSTICOS DE ENFERMAGEM NO PERÍODO PERIOPERATÓRIO: REVISÃO INTEGRATIVA
}

\author{
Nursing diagnoses in the perioperative period: integrative review
}

Diagnósticos de enfermería en el periodo perioperatorio: revisión integrativa

Aline Fritzen $^{1 *}$ (D), Luísa Pimentel Silva² (), Rita Catalina Aquino Caregnato ${ }^{3}$ (D), Graciele Fernanda da Costa Linch ${ }^{4}$ (D)

RESUMO: Objetivo: Conhecer as publicações científicas relacionadas aos diagnósticos de enfermagem (DEs) no período perioperatório do paciente cirúrgico. Método: Revisão integrativa nas bases de dados Web of Science, Scopus, Wiley Online Library e na plataforma da Revista SOBECC Nacional. Descritores utilizados na busca: "nursing diagnosis" e "surgery". Critérios de inclusão: estudos publicados entre 2014 e 2019, disponíveis em português, inglês ou espanhol, e responder à questão norteadora. Resultados: Selecionados 15 artigos, sendo oito publicados em periódicos internacionais, entretanto todos produzidos por pesquisadores brasileiros. Seis publicações identificaram os principais DEs no perioperatório, os demais pesquisaram um DE específico. Evidenciou-se o pós-operatório como o período mais pesquisado. Os estudos foram classificados conforme níveis de evidência (NE): seis com NE 4, seis com NE 5 e três com NE 6. Conclusão: O pós-operatório foi o período mais relacionado aos DEs. Apenas um estudo abordou o DE especificamente no intraoperatório. Constatou-se que a população mais estudada foi a de pacientes submetidos à cirurgia cardíaca.

Palavras-chave: Diagnóstico de enfermagem. Assistência perioperatória. Processo de enfermagem.

ABSTRACT: Objective: To assess scientific publications related to nursing diagnoses (NDs) for surgical patients in the perioperative period. Method: This is an integrative review conducted in the Web of Science, Scopus, and Wiley Online Library databases and in the platform of the National SOBECC Journal. The keywords used in the search were: "nursing diagnosis" and "surgery". The inclusion criteria were: studies published between 2014 and 2019 , available in Portuguese, English, or Spanish, and that answered the guiding question. Results: We selected 15 articles, of which 8 were published in international journals, even though all studies were produced by Brazilian researchers. Six publications identified the main NDs in the perioperative period, while the others investigated a specific ND. The postoperative period was the most studied. Studies were classified according to their level of evidence (LE): six with LE 4, six with LE 5, and three with LE 6. Conclusion: The postoperative period was the one most associated with NDs. Only one study addressed NDs specifically in the intraoperative period. The most studied population was that of patients submitted to cardiac surgery.

Keywords: Nursing diagnosis. Perioperative care. Nursing process.

RESUMEN: Objetivo: Conocer las publicaciones científicas relacionadas con los Diagnósticos de Enfermería (DEs) en el período perioperatorio del paciente quirúrgico. Método: Revisión integrativa en las bases de datos de Web of Science, SCOPUS, Wiley Online Library y la plataforma de Revista SOBECC Nacional. Descriptores utilizados en la búsqueda: “diagnóstico de enfermería” y “cirugía”. Criterios de inclusión: estudios publicados entre 2014 y 2019 , disponibles en portugués, inglés o español y que respondan a la pregunta orientadora. Resultados: Se seleccionaron 15 artículos, ocho de los cuales fueron publicados en revistas internacionales, sin embargo, todos producidos por investigadores brasileños. Seis publicaciones identificaron los principales DEs en el período perioperatorio, las otras investigaron un DE específico. El postoperatorio se convirtió en el período más investigado. Los estudios se clasificaron según niveles de evidencia (NE): seis con NE 4, seis con NE 5 y tres con NE 6. Conclusión: El postoperatorio fue el período más relacionado con la DE. Solo un estudio abordó la DE específicamente durante la operación. Se encontró que la población más estudiada fueron los pacientes sometidos a cirugía cardíaca. Palabras clave: Diagnóstico de enfermería. Atención perioperativa. Proceso de enfermería.

\footnotetext{
'Mestranda do Programa de Pós-graduação em Enfermagem pela Universidade Federal de Ciências da Saúde de Porto Alegre (UFCSPA). Supervisora de Enfermagem do Centro Cirúrgico de Transplantes e Centro Cirúrgico Ambulatorial do Hospital Dom Vicente Scherer da Irmandade Santa Casa de Misericórdia (ISCMPA) - Porto Alegre (RS), Brasil.

${ }^{2}$ Mestranda do Programa de Pós-graduação em Enfermagem pela UFCSPA. Enfermeira no Hospital Mãe de Deus - Porto Alegre (RS), Brasil.

${ }^{3}$ Doutora em Enfermagem pela Universidade Federal do Rio Grande do Sul (UFRGS). Professora adjunta IV do Departamento de Enfermagem da UFCSPA e docente do curso de Graduação em Enfermagem e do Mestrado Profissional em Enfermagem da UFCSPA - Porto Alegre (RS), Brasil.

¿Doutora em Educação pela UFRGS. Professora adjunta do Departamento de Enfermagem da UFCSPA e docente do curso de Graduação em Enfermagem e do Mestrado Profissional em Enfermagem da UFCSPA - Porto Alegre (RS), Brasil.

*Autora correspondente: afritzen10@gmail.com

Recebido: 13/02/2020 - Aprovado: 08/11/2020

https://doi.org/10.5327/Z1414-4425202100010008
} 


\section{INTRODUÇÃo}

No Brasil, o enfermeiro utiliza a sistematização da assistência de enfermagem (SAE) como metodologia científica para auxiliar a organização do cuidado, com a finalidade de aprimorar cada vez mais o atendimento das necessidades individuais dos pacientes, de forma complementar e multiprofissional ${ }^{1}$. Segundo a Associação Brasileira de Enfermeiros de Centro Cirúrgico, Recuperação Anestésica e Centro de Material e Esterilização (SOBECC), a sistematização da assistência de enfermagem perioperatória (SAEP) foi proposta antes da resolução brasileira do Conselho Federal de Enfermagem (Cofen), em 1990, quando Castellanos e Jouclas estenderam o processo de enfermagem (PE) para a assistência ao paciente cirúrgico, abrangendo os conceitos do cuidado holístico e da assistência continuada, participativa, individualizada, documentada e avaliada ${ }^{2}$.

No atendimento do paciente perioperatório, a Association of periOperative Registered Nurses (AORN) usa outro modelo padronizado, denominado de Perioperative Nursing Data Set (PNDS), que documenta os cuidados de enfermagem de maneira padronizada. Esse modelo envolve os domínios de segurança e respostas fisiológicas e comportamentais, permitindo registrar os problemas do paciente, as intervenções e os resultados de enfermagem reais ou potenciais, de modo a evidenciar a preocupação com o atendimento ${ }^{3}$.

A Resolução do Cofen $n^{\circ} 358 / 2009$ preconiza que todas as instituições de saúde onde ocorram cuidados de enfermagem devem aplicar a SAE, implementando na prática assistencial seus conhecimentos técnico-científicos, organizando o trabalho profissional em relação ao método, aos recursos humanos e instrumentos, tornando possíveis a operacionalização e a documentação do PE. Este último é dividido em cinco etapas: histórico de enfermagem, diagnóstico de enfermagem (DE), planejamento de enfermagem, implementação da assistência e avaliação da assistência prestada. Embora estejam didaticamente divididas, elas não acontecem de maneira isolada; ao contrário, estão inter-relacionadas e dão-se concomitantemente ${ }^{4}$.

A SAEP permite ao enfermeiro do centro cirúrgico (CC) qualificar a assistência aos pacientes no período perioperatório, planejando ações e promovendo melhor comunicação entre as equipes, monitorando e analisando os indicadores para propiciar a efetividade dos processos de enfermagem. Estudos ressaltam a importância da comunicação entre as equipes, a fim de contribuir para a melhoria da assistência perioperatória, desenvolvendo ações que visam orientar e avaliar as necessidades de cada paciente, resultando em um planejamento dos cuidados com qualidade durante todo o processo anestésico-cirúrgico ${ }^{5}$. O período perioperatório constitui-se de três fases: pré-operatória, intraoperatória e pós-operatória. Cada uma delas é iniciada e finalizada sequencialmente, conforme eventos que delineiam a experiência do paciente no processo da anestesia e da cirurgia ${ }^{6}$.

O enfermeiro tem a responsabilidade legal de diagnosticar as respostas humanas relacionadas à saúde ou às atividades cotidianas. Com a verificação de problemas, esse profissional poderá prever soluções e agir na prevenção de complicações e eventos adversos. Dessa maneira, pesquisadores destacam a importância da SAE visando à identificação precoce dos DEs e à resolução de possíveis complicações ${ }^{7}$.

Estudos ${ }^{8,9}$ consentem ao apresentar a relevância da identificação dos DE na clínica cirúrgica para orientar o cuidado de enfermagem, permitindo o reconhecimento prévio das necessidades manifestadas pelos pacientes, fornecendo subsídios para o estabelecimento das intervenções de enfermagem comprovadas e adequadas às necessidades individuais. Ainda, considera-se relevante investigar DE no contexto cirúrgico por ser um conhecimento específico do enfermeiro, que tem o potencial de qualificar os registros de enfermagem, melhorando a comunicação e a qualidade da assistência.

\section{OBJETIVO}

Conhecer as publicações científicas relacionadas aos DEs no período perioperatório do paciente cirúrgico.

\section{MÉTODO}

Trata-se de uma revisão integrativa da literatura que permite a identificação de achados primários acerca do assunto pesquisado, assim como o estado de conhecimento sobre ele, possibilitando a realização de uma análise crítica, reconhecendo pontos de reforço e, ao mesmo tempo, lacunas que possam ser preenchidas com novos estudos ${ }^{10,11}$.

Foram adotadas seis etapas preconizadas para a constituição da revisão integrativa ${ }^{10}$ :

- identificação do tema e definição da questão norteadora;

- estabelecimento de critérios de inclusão e exclusão;

- definição das informações a serem extraídas dos artigos;

- avaliação e categorização dos estudos incluídos;

- interpretação dos resultados;

- apresentação da síntese da revisão. 
Definiu-se como questão norteadora: o que tem sido produzido cientificamente sobre DE no período perioperatório? Com base nessa questão, foram escolhidos os descritores " $n u r s i n g$ diagnosis" e "surgery", de acordo com o Medical Subject Headings (MeSH). O operador escolhido entre os descritores foi AND, pois sua aplicação evitou a recuperação de estudos cujo assunto tangenciasse outras áreas de conhecimento não objetivadas para a análise em questão. Os critérios de inclusão foram: estudos publicados de 2014 até setembro de 2019; disponibilidade em português, inglês ou espanhol; responder à questão norteadora; e que contivessem em seu título um dos descritores da busca ou termos relacionados: "surgical", "nursing outcomes", "nursing interventions and outcomes", "operative" e "“"postoperative". Como um dos critérios de inclusão foi o ano de publicação, foram selecionados apenas artigos a partir do ano de 2014, visto que o propósito da pesquisa foi elencar publicações recentes.

A busca pelos estudos ocorreu nas bases de dados Web of Science, Scopus e Wiley Online Library. Complementou-se a busca na plataforma da Revista SOBECC Nacional, por ser esta uma revista técnica da área estudada de grande importância. Nas bases de dados foi empregada a seguinte estratégia associativa: "nursing diagnosis" AND “surgery”, selecionando sempre o campo mais genérico de busca em todas elas. Na plataforma da Revista SOBECC Nacional, em que há divulgação técnico-científica da SOBECC, utilizou-se somente o descritor "nursing diagnosis", pois a temática das publicações dessa base já é voltada à área cirúrgica.

As buscas nas bases de dados resultaram em 98 artigos. Os títulos das publicações obtidas foram lidos, e eliminaram-se aquelas que não atendiam aos critérios de inclusão, restando 30 artigos. Em seguida, estes foram filtrados mediante a leitura dos resumos, reduzindo-se a amostra para 24 artigos. As publicações foram lidas na íntegra, chegando-se à seleção final de 15 artigos, excluindo-se uma publicação repetida, encontrada em duas bases de dados. Na Figura 1, apresenta-se o fluxograma para a seleção dos artigos incluídos no estudo.

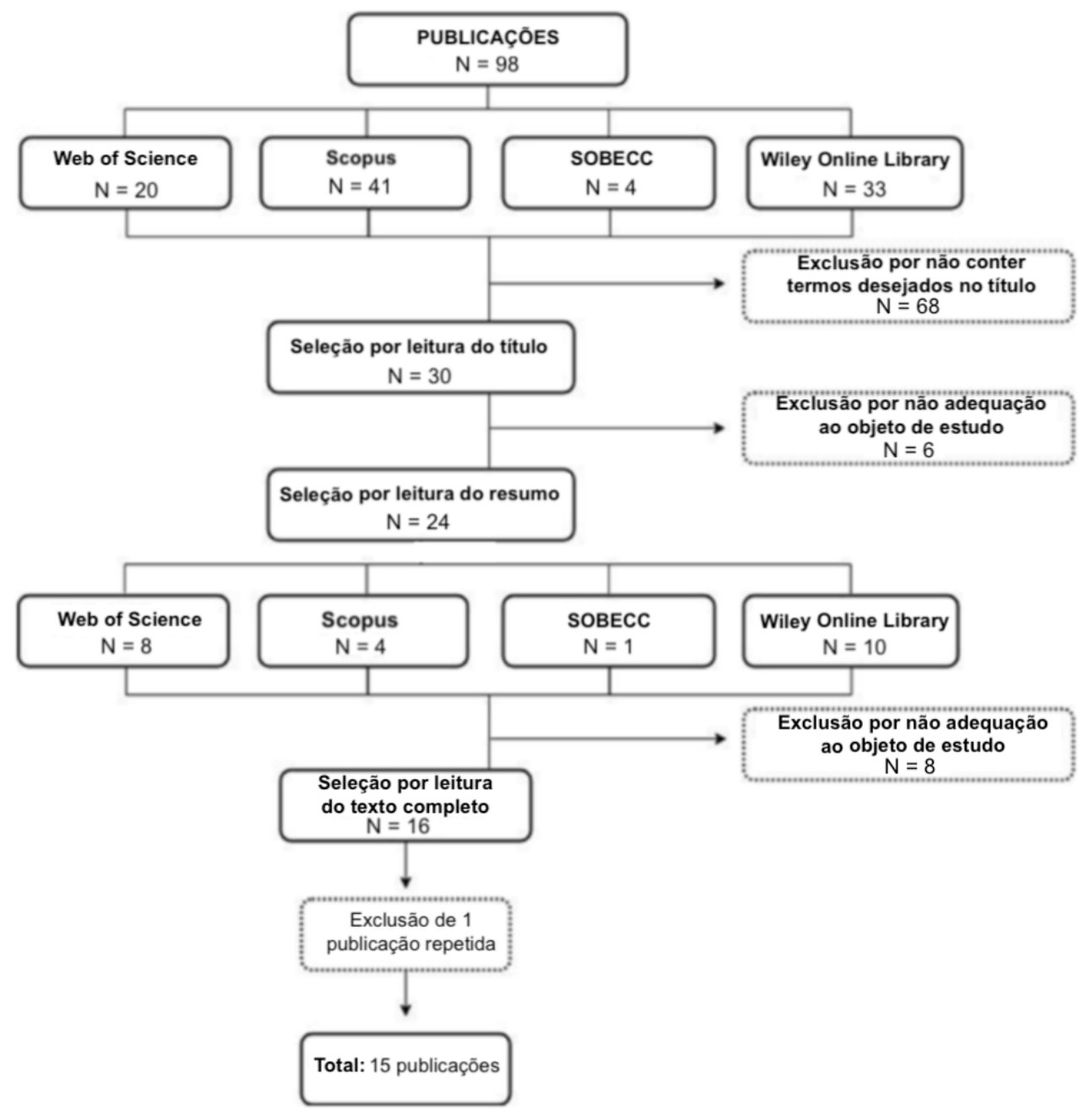

Figura 1. Fluxograma do processo de seleção dos artigos. 
A coleta de dados realizou-se em setembro de 2019 , e as publicações obtidas foram organizadas para análise em um banco de dados com os seguintes itens: título, base de dados, periódico, ano, idioma, país de origem, objetivo, método, grau de evidência e período do perioperatório abordado no artigo.

Posteriormente, os artigos foram avaliados quanto ao nível de evidência (NE), o qual caracteriza a maneira como as evidências são classificadas segundo a forma hierárquica e de acordo com a abordagem metodológica adotada ${ }^{10}$.

\section{RESULTADOS}

Evidenciaram-se, na amostra selecionada, oito artigos publicados em periódicos internacionais, entretanto todos foram produzidos por pesquisadores brasileiros. Em relação ao ano de publicação, o pico maior de produtividade ocorreu em 2015 , com cinco publicações. Identificou-se um gap sem publicações sobre o tema entre 2016 e 2017 . Nos demais anos houve três publicações em 2014, quatro em 2018 e três em 2019.

Apresentam-se os artigos em dois quadros, conforme seus objetivos. No Quadro 1, encontram-se seis artigos que, haja vista uma população específica estudada, abordaram os principais DEs relacionados. No Quadro 2 foram agrupados os artigos com objetivos diversos quanto a DEs específicos previamente elencados.

No total, foram citados 105 DEs, contudo 39 deles se repetiram, o que significa que foram encontrados 66 DEs diferentes, sendo 42 reais e 24 de risco. Com esse resultado, confeccionaram-se dois gráficos (Gráficos 1 e 2) para os dez DEs

Quadro 1. Artigos selecionados, que abordam uma população específica, quanto a ano de publicação, objetivo, diagnósticos de enfermagem estudados e nível de evidência.

\begin{tabular}{|c|c|c|c|}
\hline Ano & Objetivo & DEs estudados & NE \\
\hline $2015^{7}$ & $\begin{array}{l}\text { Identificar as respostas } \\
\text { humanas apresentadas pelos } \\
\text { pacientes no pós-operatório } \\
\text { de cirurgia bariátrica } \\
\text { classificadas na classe de } \\
\text { respostas cardiovasculares/ } \\
\text { pulmonares dos DEs }\end{array}$ & $\begin{array}{l}\text { - Risco de perfusão gastrintestinal ineficaz; } \\
\text { - Risco de intolerância à atividade; } \\
\text { - Perfusão tissular periférica ineficaz; } \\
\text { - Risco de choque; } \\
\text { - Débito cardíaco diminuído; } \\
\text { - Risco de perfusão tissular cardíaca diminuída; } \\
\text { - Intolerância à atividade; } \\
\text { - Risco de perfusão tissular cerebral ineficaz; } \\
\text { - Ventilação espontânea prejudicada; } \\
\text { - Risco de perfusão renal ineficaz; } \\
\text { - Padrão respiratório ineficaz; } \\
\text { - Resposta disfuncional ao desmame ventilatório; } \\
\text { - Risco de sangramento. }\end{array}$ & 4 \\
\hline $2015^{8}$ & $\begin{array}{l}\text { Identificar DE em pacientes } \\
\text { no pós-operatório de cirurgia } \\
\text { cardíaca }\end{array}$ & $\begin{array}{l}\text { - Risco de perfusão renal ineficaz; } \\
\text { - Risco de perfusão tissular cardíaca diminuída; } \\
\text { - Risco de sangramento; } \\
\text { - Risco de lesão no posicionamento perioperatório; } \\
\text { - Integridade da pele prejudicada; } \\
\text { - Risco de quedas; } \\
\text { - Risco de trauma vascular; } \\
\text { - Risco de constipação; } \\
\text { - Risco de desequilíbrio eletrolítico; } \\
\text { - Risco de nível instável de glicose no sangue; } \\
\text { - Risco de confusão aguda; } \\
\text { - Risco de choque; } \\
\text { - Risco de infecções; } \\
\text { - Risco de sofrimento espiritual; } \\
\text { - Risco de religiosidade prejudicada; } \\
\text { - Mobilidade física prejudicada; } \\
\text { - Mobilidade prejudicada no leito; } \\
\text { - Disposição para processos familiares melhorados; } \\
\text { - Disposição para autoconceito melhorado; }\end{array}$ & 5 \\
\hline
\end{tabular}


Quadro 1. Continuação.

\section{Ano} Objetivo

Identificar DE em pacientes no pós-operatório de cirurgia cardíaca

Propor diagnósticos, resultados e intervenções de $2018^{12} \quad$ enfermagem em pacientes no pós-operatório de cirurgia ortognática
Identificar os principais DEs em idosos no pós-operatório de cirurgias urológicas
- Conforto prejudicado;

- Motilidade gastrintestinal disfuncional;

- Deambulação prejudicada;

- Dor aguda;

- Recuperação cirúrgica retardada;

- Distúrbio do padrão do sono;

- Medo;

- Fadiga;

- Padrão respiratório ineficaz;

- Insônia;

- Ventilação espontânea prejudicada;

- Envolvimento nas atividades diminuído;

- Comunicação verbal prejudicada;

- Ansiedade;

- Controle ineficaz da saúde;

- Controle ineficaz de saúde familiar;

- Risco de perfusão tissular periférica ineficaz;

- Síndrome do estresse por mudança;

- Hipertermia;

- Termorregulação ineficaz;

- Volume de líquidos deficiente;

- Constipação;

- Troca gasosa prejudicada;

- Diminuição do débito cardíaco;

- Autonegligência;

- Náusea;

- Deglutição prejudicada;

- Nutrição desequilibrada: menor que as necessidades do corpo;

- Interação social prejudicada;

- Membrana mucosa oral comprometida.

- Controle ineficaz da saúde;

- Conhecimento deficiente;

- Comunicação verbal prejudicada;

- Risco de dignidade humana comprometida;

- Risco de baixa autoestima situacional;

- Ansiedade;

- Risco de infecção;

- Membrana mucosa oral comprometida;

- Dor aguda.

- Integridade da pele prejudicada;

- Intolerância à atividade;

- Dor aguda;

- Conhecimento deficiente;

- Nutrição desequilibrada: menor que as necessidades corporais;

- Risco de constipação;

- Fadiga;

- Mobilidade física prejudicada;

- Distúrbio no padrão de sono;

- Risco de infecção;

- Risco de volume de líquidos deficiente;

- Risco de religiosidade prejudicada. 
Quadro 1. Continuação.

\begin{tabular}{|c|c|c|c|}
\hline Ano & Objetivo & DEs estudados & NE \\
\hline $2015^{14}$ & $\begin{array}{l}\text { Identificar a frequência dos } \\
\text { DEs em pacientes de uma } \\
\text { clínica cirúrgica }\end{array}$ & $\begin{array}{l}\text { - Risco de infecção; } \\
\text { - Integridade da pele prejudicada; } \\
\text { - Risco de constipação; } \\
\text { - Ansiedade; } \\
\text { - Dor aguda; } \\
\text { - Risco de sangramento; } \\
\text { - Recuperação cirúrgica retardada; } \\
\text { - Motilidade gastrintestinal disfuncional; } \\
\text { - Risco de síndrome de estresse por mudanças; } \\
\text { - Risco de glicose; } \\
\text { - Conforto prejudicado; } \\
\text { - Risco de integridade da pele prejudicada; } \\
\text { - Medo; } \\
\text { - Nutrição desequilibrada: menor que as necessidades corporais; } \\
\text { - Risco situacional de baixa autoestima; } \\
\text { - Risco de volume de líquidos desequilibrado; } \\
\text { - Perturbação da imagem corporal. }\end{array}$ & 5 \\
\hline $2015^{15}$ & $\begin{array}{l}\text { Identificar o perfil dos DEs } \\
\text { nos pacientes transplantados } \\
\text { cardíacos em pós-operatório } \\
\text { mediato, com base na } \\
\text { taxonomia Il da NANDA, } \\
\text { e discuti-lo à luz dos } \\
\text { pressupostos de Horta e da } \\
\text { literatura científica }\end{array}$ & $\begin{array}{l}\text { - Mobilidade no leito prejudicada; } \\
\text { - Proteção ineficaz; } \\
\text { - Deambulação prejudicada; } \\
\text { - Integridade tecidual prejudicada; } \\
\text { - Nutrição desequilibrada: menor que as necessidades corporais; } \\
\text { - Débito cardíaco diminuído; } \\
\text { - Dor aguda; } \\
\text { - Troca de gases prejudicada; } \\
\text { - Padrão de respiração ineficaz; } \\
\text { - Eliminação urinária prejudicada; } \\
\text { - Risco de infecção; } \\
\text { - Risco de constipação. }\end{array}$ & 4 \\
\hline
\end{tabular}

Quadro 2. Artigos selecionados que discutem um DE no período perioperatório, quanto ao ano de publicação, objetivo, DE estudado e NE.

\section{Ano}

Objetivos

Selecionar resultados do NOC para avaliar a integridade do tecido prejudicado em

$2019^{16}$ pacientes submetidos a cirurgias ortopédicas e desenvolver definições conceituais e operacionais para seus indicadores

201917

Avaliar os benefícios das intervenções NIC em pacientes pós-operatórios com depuração ineficaz das vias aéreas.

$2019^{18}$

Selecionar e refinar os resultados e indicadores do NOC para o diagnóstico de risco de lesão por posicionamento perioperatório

$2019^{19}$

Avaliar a cicatrização de feridas cirúrgicas (SW) em pacientes ortopédicos com integridade tecidual prejudicada de acordo com o NOC

$2018^{20}$

Identificar os fatores de risco associados aos casos de sangramento excessivo em pacientes submetidos à cirurgia cardíaca com circulação extracorpórea

$2018^{21}$

Esclarecer o conceito de risco de recuperação cirúrgica tardia e definir os referentes empíricos para identificação de fatores preditivos de atraso na recuperação

$2015^{22}$

Verificar a acurácia das características definidoras do diagnóstico de recuperação cirúrgica tardia em pacientes após o quinto dia de pós-operatório

$2015^{23}$ Analisar a precisão das características definidoras das trocas gasosas comprometidas

$2014^{24}$ Comparar a ocorrência do DE de recuperação cirúrgica tardia na população adulta e idosa
DE estudado

- Integridade tecidual prejudicada

- Desobstrução ineficaz das vias aéreas

- Risco de lesão por posicionamento perioperatório

- Integridade tecidual prejudicada

- Risco de sangramento

- Risco para recuperação cirúrgica retardada

- Recuperação cirúrgica retardada

- Trocas gasosas prejudicadas

- Recuperação cirúrgica retardada

DE: diagnóstico de enfermagem; NE: nível de evidência; NOC: Nursing Outcomes Classification; NIC: Nursing Interventions Classification. 


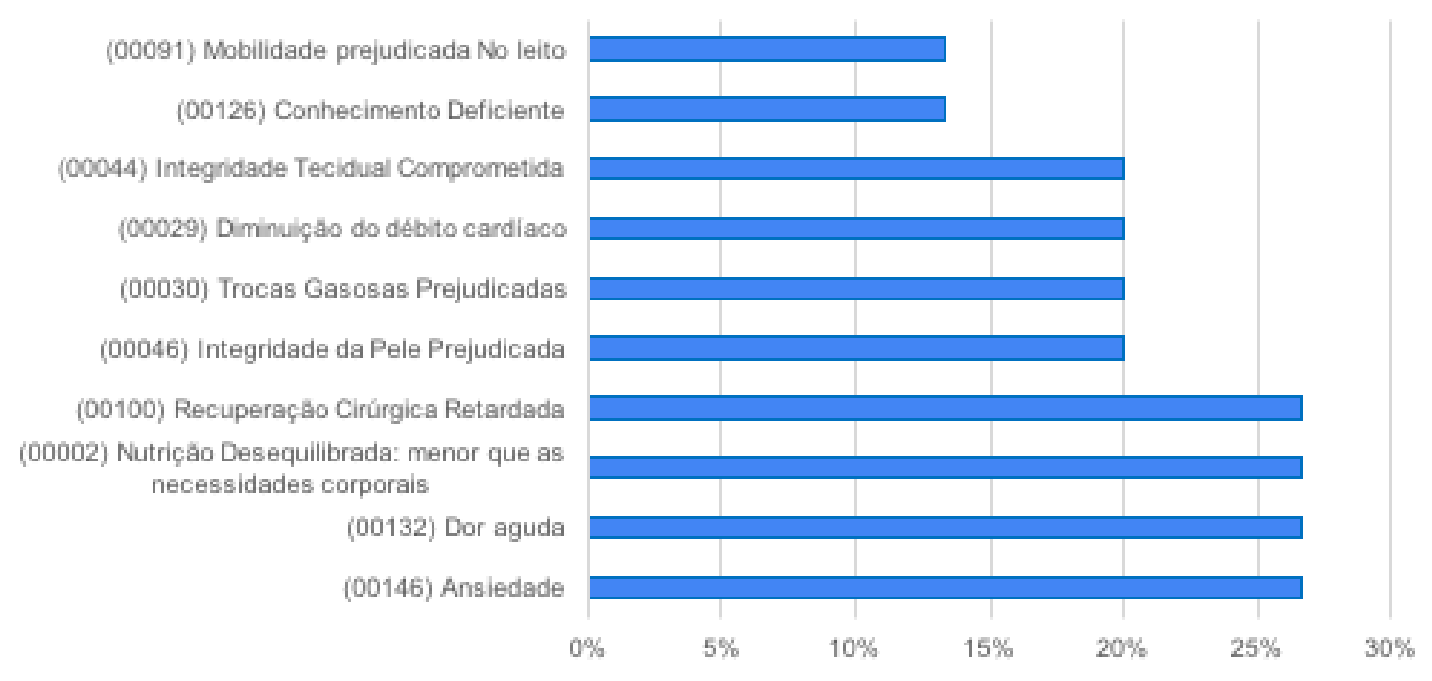

Gráfico 1. Diagnósticos de enfermagem reais elencados nos artigos.

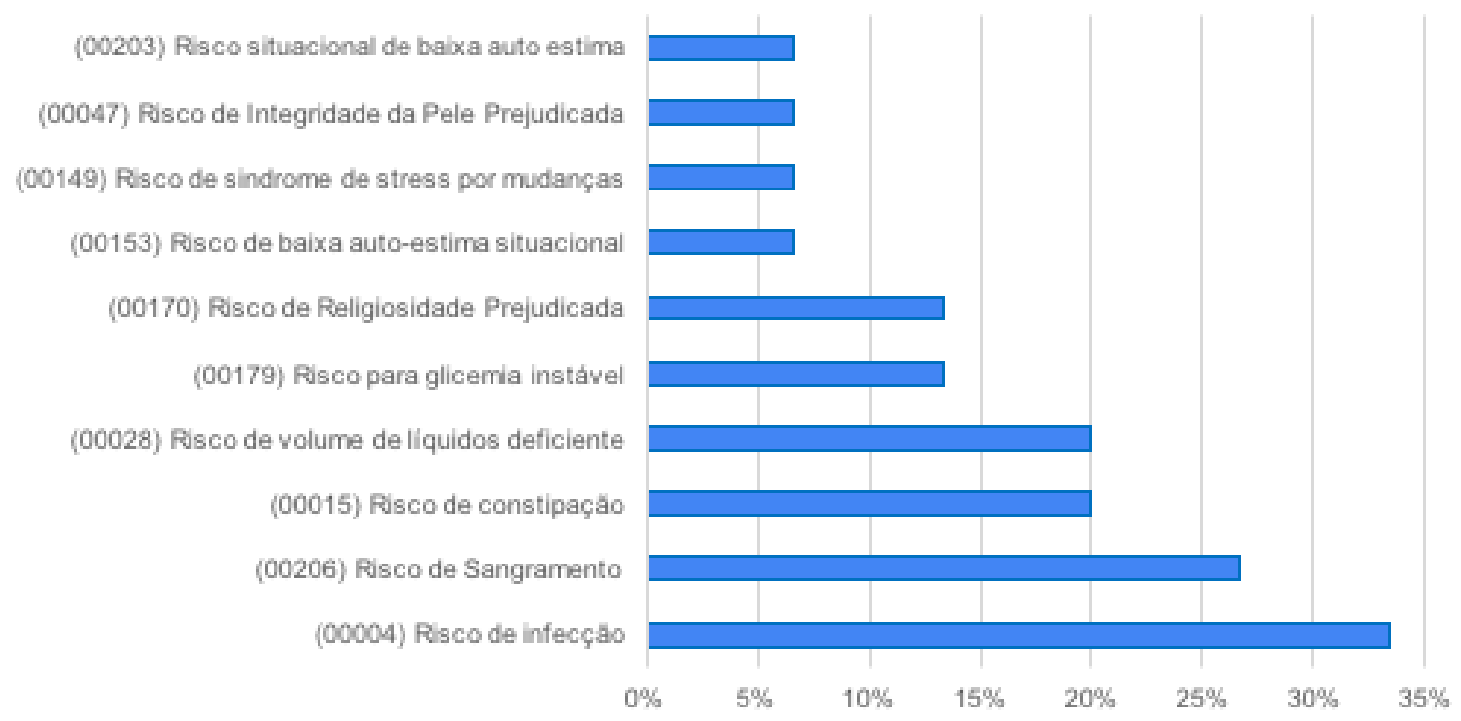

Gráfico 2. Diagnósticos de enfermagem de risco elencados nos artigos.

reais e de risco mais prevalentes nos estudos. Para cada um, foi apresentado o percentual de aparição no que se refere às 15 publicações estudadas. O DE de ansiedade, por exemplo, foi elencado em quatro das 15 publicações, representando $26,67 \%$ de recorrência.

\section{DISCUSSÃO}

A análise das publicações selecionadas possibilitou demonstrar que a maioria dos estudos (dez artigos) sobre a temática investigada aborda o pós-operatório em diferentes cenários de pacientes cirúrgicos. Ao mesmo tempo, os estudos discorrem sobre as várias etapas do $\mathrm{PE}$, como $\mathrm{DE}$, intervenções e resultados.

O referencial teórico das necessidades humanas básicas de Wanda de Aguiar Horta foi aplicado em dois estudos ${ }^{12-15}$ para elencar DEs em pacientes pós-operatórios. Um deles ${ }^{12}$ identificou nove DEs em pacientes no pós-operatório de cirurgia ortognática, relacionados às necessidades psicobiológicas e psicossociais. Outro estudo ${ }^{15}$, realizado com pacientes transplantados de cirurgia cardíaca, detectou 12 DEs, sendo 
dez DEs reais e dois DEs potenciais, todos pertencentes à necessidade humana básica psicobiológica. Constatou-se não terem sido identificados DEs para necessidades psicossociais nem psicoespirituais.

Dois estudos ${ }^{13,14}$ efetuados com diferentes populações, mas com o mesmo objetivo, identificaram os principais DEs no pós-operatório aplicáveis a cada uma das populações de pacientes. Em um deles ${ }^{13}$ foram revisados os prontuários de cem idosos em pós-operatório de cirurgias urológicas e foram apurados 13 DEs, sendo 10 DEs reais e três DEs de risco. No outro ${ }^{14}$, com amostra de 99 pacientes em pós-operatório de cirurgia geral, foram sinalizados $17 \mathrm{DEs}$, sendo nove DEs reais e oito DEs de risco. Em ambos os estudos ${ }^{13,14}$, os achados permitiram direcionar os cuidados de enfermagem priorizando a clínica do paciente, com ações eficazes e imediatas para a resolução dos problemas.

A importância da SAEP e a necessidade de implementar protocolos institucionais que facilitem os cuidados de enfermagem prestados a cada perfil de paciente foram ressaltadas também em um estudo ${ }^{7}$ feito com uma população de pacientes pós-operatórios de cirurgia bariátrica. Esse estudo teve como objetivo identificar respostas humanas apresentadas pelos pacientes classificadas na classe de respostas cardiovasculares/pulmonares dos DEs. Foram elencados 13 DEs, possibilitando direcionar os cuidados de enfermagem e reduzir as complicações pós-operatórias.

Constatou-se que a população mais estudada foi a de pacientes submetidos à cirurgia cardíaca. Um dos estudos ${ }^{20}$ buscou apontar fatores de risco para sangramento após cirurgia cardíaca com circulação extracorpórea (CEC) e concluiu que as variáveis associadas ao sangramento excessivo foram: índice de massa corporal (IMC) menor que $26,35 \mathrm{~kg} / \mathrm{m}^{2}$, tempo de CEC superior a 90 minutos, temperatura esofágica menor que $32^{\circ} \mathrm{C}$, acidose metabólica e tempo de tromboplastina superior a 40 segundos. Tais variáveis podem ser indicadores clínicos de natureza operacional para melhor caracterização do fator de risco "regime de tratamento" e aprimoramento do conhecimento relacionado à coagulopatia induzida pela CEC.

Outros dois estudos ${ }^{8,23}$ versavam sobre pós-operatório de cirurgia cardíaca em unidades de terapia intensiva. Um deles avaliou prontuários de 26 pacientes, em que especialistas coletaram DEs, características definidoras e fatores relacionados, resultando em $34 \mathrm{DEs}$ reais e $15 \mathrm{DEs}$ de risco, estabelecidos de acordo com a North American Nursing Diagnosis Association (NANDA) ${ }^{8}$. Na outra pesquisa ${ }^{23}$, houve envolvimento de uma amostra de 93 pacientes. Os dados foram coletados por três enfermeiros, para analisar a precisão das características definidoras para o DE "troca gasosa comprometida”. Em ambos os estudos ${ }^{8,23}$, ficou clara a relevância do conhecimento da enfermagem sobre a identificação precoce dos sinais e sintomas (características definidoras), a fim de escolher o DE específico para cada perfil de paciente, orientar os cuidados de enfermagem (intervenções) e subsidiar o desenvolvimento de intervenções adequadas às necessidades individuais dos pacientes.

No que tange às intervenções de enfermagem, um estudo ${ }^{17}$ teve o objetivo de avaliar os benefícios das intervenções Nursing Interventions Classification (NIC) em pacientes pós-operatórios com $\mathrm{DE}$ "desobstrução ineficaz das vias aéreas”. Uma amostra de 101 pacientes foi avaliada com base no resultado de enfermagem "padrão respiratório: permeabilidade das vias aéreas". Por um lado, concluiu-se que intervenções como técnicas de respiração, melhora da tosse, assistência ventilatória e manejo das vias aéreas contribuíram para expulsão da secreção e houve melhorias expressivas da permeabilidade das vias aéreas, força e recuperação dos pacientes. Seis indicadores de perviedade das vias aéreas melhoraram significativamente ao longo do estudo. Por outro lado, alguns pacientes não melhoraram após intervenções, o que pode estar associado a fatores intrínsecos do paciente, características clínicas e variáveis cirúrgicas.

Dois estudos ${ }^{22,24}$ trataram especificamente do DE "recuperação cirúrgica retardada". Curiosamente, os dois avaliaram 72 pacientes cirúrgicos após o quinto dia de pós-operatório e, em ambos os artigos, o primeiro autor é o mesmo. Em um dos estudos ${ }^{24}$, a população foi dividida em sujeitos adultos e idosos, com o objetivo de comparar a ocorrência desse DE entre os grupos. Os achados indicaram que a prevalência foi ligeiramente maior nos idosos, por causa da dificuldade de movimentação, dependência de autocuidado e percepção da necessidade de maior tempo para a recuperação, necessitando de cuidados de enfermagem específicos ao processo de envelhecimento humano. A diferenciação da assistência entre o paciente adulto e o idoso pode favorecer o cuidado perioperatório no tempo desejado.

Outro estudo ${ }^{22}$ buscou verificar a acurácia das características definidoras do mesmo DE "recuperação cirúrgica retardada”. A identificação dos DEs foi baseada no julgamento clínico de duas enfermeiras doutoras. Sete características apresentaram valores preditivos positivos elevados: adiamento da retomada das atividades de trabalho/emprego, fadiga, percepção de necessidade de maior tempo para se recuperar, necessidade de ajuda para concluir o autocuidado, relato 
de desconforto, evidência de interrupção da cicatrização da área cirúrgica e dificuldade de movimentação. $O$ único fator do estudo associado ao diagnóstico foi a infecção pós-operatória no local cirúrgico.

Um estudo ${ }^{21}$ relacionado ao DE "risco para recuperação cirúrgica retardada” desenvolveu uma análise para esclarecer o conceito desse diagnóstico e definir os referentes empíricos para identificar fatores preditivos de atraso na recuperação. Foram selecionados enfermeiros especialistas em enfermagem cirúrgica e em DE, segundo a taxonomia NANDA-I. Por meio de uma revisão integrativa da literatura, os autores não encontraram nenhum estudo que abordasse diretamente o diagnóstico, mostrando lacunas na exploração do diagnóstico estudado, que agrupa uma série de fatores de risco nas melhores evidências e estabelece referências empíricas para instrumentação e avaliação, o que pode ajudar a orientar enfermeiros a obter os resultados esperados na prática.

Nos artigos selecionados, dois estudos ${ }^{16,18}$ foram de validação por consenso de especialistas, buscando selecionar e refinar os resultados e indicadores da Nursing Outcomes Classification (NOC) para os DEs "risco de lesão por posicionamento perioperatório" e "integridade tecidual prejudicada". Ambos possibilitaram selecionar os resultados e os indicadores mais relevantes a serem medidos para esses diagnósticos na prática clínica.

Ainda acerca da NOC, pesquisadores ${ }^{19}$ avaliaram a cicatrização de feridas cirúrgicas em 24 pacientes ortopédicos de artroplastia de quadril, com DE "integridade tissular prejudicada", estabelecido pela avaliação de duas enfermeiras e documentado nos registros eletrônicos de saúde, pela aplicação dos instrumentos de coleta de dados, o que significa realizar entrevistas com pacientes e avaliar a ferida operatória.
Ao comparar o primeiro e o último dia, os pacientes tiveram melhora progressiva em três indicadores: aproximação da pele, drenagem e sinais inflamatórios, e odor desagradável.

\section{CONCLUSÃO}

Ao conhecer a produção científica relacionada aos DEs no período perioperatório do paciente, constatou-se o pós-operatório como o período mais pesquisado, embora a maioria dos estudos tratasse o tema com um olhar direcionado a todo o processo anestésico-cirúrgico. As pesquisas mostram DEs mais prevalentes em pacientes cirúrgicos englobando as três etapas do processo perioperatório, entretanto apenas um estudo abordou o DE "risco de lesão por posicionamento perioperatório" especificamente no intraoperatório. Comprovou-se que a população mais estudada foi a de pacientes submetidos à cirurgia cardíaca.

Chamou a atenção que todos os artigos selecionados foram produzidos por enfermeiros brasileiros, mesmo os publicados em periódicos internacionais, demonstrando o interesse nacional sobre esse tema.

Sugere-se que outras pesquisas sobre os DEs no processo perioperatório sejam realizadas, explorando DEs atuais, como, por exemplo, "risco de infecção no sítio cirúrgico", aprovado em 2016, mas que não foi citado nas pesquisas revisadas. Cabe aos enfermeiros buscarem conhecimentos e aperfeiçoamento constantes, a fim de contribuir para o aprimoramento do processo assistencial. Para que o enfermeiro se sinta seguro em relação ao julgamento e raciocínio clínico, o PE precisa ser trabalhado continuamente em formações e atividades educativas.

\section{REFERÊNCIAS}

1. Viana VO, Pires PS. Validação de Instrumento de Sistematização da Assistência de Enfermagem. Rev Enferm Atenção Saúde [Internet]. 2014 [acesso em 10 set. 2019];3(2):64-75. Disponivel em: http://seer. uftm.edu.br/revistaeletronica/index.php/enfer/article/view/1021/884. https://doi.org/10.18554/

2. Associação Brasileira de Enfermeiros de Centro Cirúrgico, Recuperação Anestésica e Centro de Material e Esterilização (SOBECC). Diretrizes de práticas em enfermagem cirúrgica e processamento de produtos para a saúde. $7^{a}$ ed. São Paulo: SOBECC/Barueri: Manole; 2017.

3. Association of periOperative Registered Nurses (AORN). Guidelines for perioperative practice. Denver: AORN; 2018.
4. Conselho Federal de Enfermagem (COFEn). Resolução n 358 , de 15 de outubro de 2009. Dispõe sobre a Sistematização da Assistência de Enfermagem e a implementação do Processo de Enfermagem em ambientes, públicos ou privados, em que ocorre o cuidado profissional de enfermagem, e dá outras providências [Internet]. Brasil: Portal COFEn; 2009 [acesso em 10 set. 2019]. Disponivel em: http://www.cofen.gov.br/resoluocofen-3582009_4384.html

5. Jost MT, Viegas K, Caregnato RCA. Sistematização da assistência de enfermagem perioperatória na segurança do paciente: revisão integrativa. Rev SOBECC. 2018;23(4):218-25. https://doi.org/10.5327/ Z1414-4425201800040009 
6. Suarni L, Nurjannah I, Apriyani H. Nursing and collaborative diagnoses on perioperative patients with and without using six steps of diagnostic reasoning methods. Int J Res Med Sci [Internet]. 2015 [acesso em 15 set. 2019];3(1):97-103. Disponível em: https://www.researchgate.net/ publication/288040348_Nursing_and_collaborative_diagnoses_on_ perioperative_patients_with_and_without_using_six_steps_of_diagnostic reasoning_methods. https://doi.org/10.18203/2320-6012.jirms20151528

7. Barros LM, Moreira RAN, Frota NM, Caetano JA. Identificação dos diagnósticos de enfermagem da classe de respostas cardiovasculares/ pulmonares em pacientes submetidos à cirurgia bariátrica. Aquichan [Internet]. 2015 [acesso em 23 set. 2019];15(2):200-9. Disponível em: http://www.scielo.org.co/scielo.php?script=sci_arttext\&pid=S165759972015000200004\&lng=en. http://doi.org/10.5294/aqui.2015.15.2.4

8. Ribeiro CP, Silveira CO, Benetti ERR, Gomes JS, Stumm EMF. Nursing diagnoses for patients in the postoperative period of cardiac surgery. Rev Rede Enferm Nordeste [Internet]. 2015 [acesso em 17 set. 2019];16(2):159-67. Disponível em: https:// www.redalyc.org/pdf/3240/324038465004_2.pdf. http://doi. org/10.15253/2175-6783.2015000200004

9. Novaes ES, Torres MM, Oliva APV. Diagnósticos de enfermagem em clínica cirúrgica. Acta Paul Enferm. 2015;28(1):26-31. https://doi. org/10.1590/1982-0194201500006

10. Soares CB, Hoga LAK, Peduzzi M, Sangaleti C, Yonekura T, Silva DRAD. Revisão integrativa: conceitos e métodos utilizados na enfermagem. Rev Esc Enferm USP. 2014;48(2):335-45. https://doi.org/10.1590/ S0080-6234201400002000020

11. Souza MT, Silva MD, Carvalho R. Revisão integrativa: o que é e como fazer. Einstein. 2010;8(1 Pt 1):102-6. http://doi.org/10.1590/ s1679-45082010rw1134

12. Assis GLC, Sousa CS, Turrini RNT, Poveda VB, Silva RCG. Proposal of nursing diagnoses, outcomes and interventions for postoperative patients of orthognathic surgery. Rev Esc Enferm USP. 2018;52:e03321. https://doi.org/10.1590/s1980-220x2017025303321

13. Lima WG, Nunes SFL, Alvarez AM, Valcarenghi RV, Bezerra MLR. Main nursing diagnoses in hospitalized elderly people who underwent urological surgery. Rev Rede Enferm Nordeste [Internet]. 2015 [acesso em 22 set 2019];16(1):72-80. Disponível em: https://www. researchgate.net/publication/275243464_Main_nursing_diagnoses_ in_hospitalized_elderly_people_who_underwent_urological_surgery. https://doi.org/10.15253/2175-6783.2015000100010

14. Vasconcelos AC, Castro CGA, Silva DF, Sousa VJ. Frequency of nursing diagnoses in a surgical clinic. Rev Rede Enferm Nordeste [Internet]. 2015 [acesso em 22 set. 2019];16(6):826-32. Disponível em: https://www.researchgate.net/publication/287800018_ Frequency_of_nursing_diagnoses_in_a_surgical_clinic. https:// doi.org/10.15253/2175-6783.2015000600008

15. Matos SS, Ferraz AF, Guimarães GL, Goveia VR, Mendoza IYQ, Silqueira SMF, et al. Heart transplanted patients in mediate postoperative period: nursing diagnoses based on horta assumptions. Rev SOBECC. 2015;20(4):228-35. https://doi.org/10.5327/Z1414-4425201500040007

16. Silva MB, Barreto LNM, Panato BP, Engelman B, Figueiredo MS, Rodríguez-Acelas AL, et al. Clinical indicators for evaluation of outcomes of impaired tissue integrity in orthopedic patients: consensus study. Int J Nurs Knowl [lnternet]. 2019 [acesso em 3 out. 2019];30(2):81-6. Disponível em: https://www.ncbi.nlm.nih.gov/ pubmed/29489065. https://doi.org/10.1111/2047-3095.12204

17. Silva LFM, Pascoal LM, Nunes SFL, Freire VECS, Almeida AGA, Gontijo PVC, et al. Ineffective airway clearance in surgical patients: evaluation of nursing interventions and outcomes. Int J Nurs Knowl [Internet]. 2019 [acesso em 28 set. 2019];30(4):251-6. Disponível em: https://www.ncbi.nlm.nih.gov/pubmed/30916452. https://doi. org/10.1111/2047-3095.12242

18. Lima LB, Cardozo MCE, Bernardes DS, Rabelo-Silva ER. Nursing outcomes for patients with risk of perioperative positioning injury. Int J Nurs Knowl [Internet]. 2019 [acesso em 2 out. 2019];30(2):1149. Disponível em: https://www.ncbi.nlm.nih.gov/pubmed/29659186. https://doi.org/10.1111/2047-3095.12209

19. Barreto LNM, Silva MBD, Engelman B, Figueiredo MS, Rodríguez-Acelas AL, Cañon-Montañez W, et al. Evaluation of surgical wound healing in orthopedic patients with impaired tissue integrity according to nursing outcomes classification. Int J Nurs Knowl [Internet]. 2019 [acesso em 5 out. 2019];30(4):228-33. Disponível em: https://www.ncbi.nlm.nih. gov/pubmed/30479065. https://doi.org/10.1111/2047-3095.12233

20. Braga DV, Brandão MAG. Diagnostic evaluation of risk for bleeding in cardiac surgery with extracorporeal circulation. Rev Latino-Am Enferm. 2018;26:e3092. https://doi.org/10.1590/1518-8345.2523.3092

21. Rembold SM, Santana RF, Souza PA, Schwartz SMOX. Nursing diagnosis risk for delayed surgical recovery (00246): concept clarification and definition of empirical referents. Int J Nurs Knowl [Internet]. 2018 [acesso em 28 set. 2019];29(4):263-8. Disponível em: https://www.ncbi.nlm.nih.gov/pubmed/28544813. https://doi. org/10.1111/2047-3095.12176

22. Santana RF, Lopes MVO. Measures of clinical accuracy and indicators of the nursing diagnosis of delayed surgical recovery. Collegian [Internet]. 2015 [acesso em 29 set. 2019];22(3):275-82. Disponível em: https://www.collegianjournal.com/article/S1322-7696(14)00019-5/ references

23. Sousa VE, Pascoal LM, Matos TF, Nascimento RV, Chaves DB, Guedes NG, et al. Clinical Indicators of Impaired Gas Exchange in Cardiac Postoperative Patients. Int J Nurs Knowl [Internet]. 2015 [acesso em 25 set. 2019];26(3):141-6. Disponivel em: https://www.ncbi.nlm.nih. gov/pubmed/25257137. https://doi.org/10.1111/2047-3095.12061

24. Santana RF, Amaral DM, Pereira SK, Delphino TM, Cassiano KM. The occurrence of the delayed surgical recovery nursing diagnosis among adults and the elderly. Acta Paul Enferm. 2014;27(1):35-9. https://doi.org/10.1590/1982-0194201400008 